\title{
DER BEGINN DER FREISINGISCHEN HERRSCHAFT IN GROSS-ENZERSDORF IM HINBLICK AUF DIE ENTSTEHUNG DES KIRCHLICHEN LEBENS: VON DEN ANFÄNGEN BIS ZU BISCHOF BERTHOLD VON WEHINGEN
}

\section{Zusammenfassung}

Der vorliegende Artikel beschäftigt sich mit der Gründung von Ort und Pfarre Groß-Enzersdorf (Enzersdorf im Text). Große Bedeutung erlangte dabei das Hochstift Freising mit seinen Bischöfen, die sowohl das kirchliche wie auch kommunale Leben prägten. In diesem Artikel befasst sich der Autor mit der Entstehung von Enzersdorf, beginnend mit der Schenkung Kaiser Heinrichs II. bis hin zu Bischof Berthold von Wehingen, der die Ortschaft bis heute prägt.

Schlüsselworte: Pfarrer, Bischof Berthold von Wehingen, Hochstift, Freising, Passau, Weihenstephan, Urkunde, Leopold, Kagran.

Am 14. November 1021 beglaubigte Kaiser Heinrich II., der letzte Kaiser aus dem Geschlecht der Ottonen (973 oder 978-1024), in Mehringen ${ }^{1}$ bei Alt-Ötting im Andenken an König Otto (III.), seinen verstorbenen Vater, dem Kloster Weihenstephan in Bayern, dessen Gründung vom Heiligen Korbinian gefördert wurde, einen Teil der Insel Sahsonaganc ${ }^{2}$, lat. insula Sahsonaganc, östlich von

* Ks. Arkadiusz Borowski - Promotion in Theologie, Erzdiözese Wien;

e-mail: arkadiusz.borowski@katholischekirche.at

https://oricd.org/ 0000-0003-4889-267X

${ }^{1}$ Mehring ist heute eine Gemeinde im oberbayerischen Landkreis Altötting.

${ }^{2}$ Zum besseren Verständnis: Das Schenkungsgut, die Insel Sahsonaganc, liegt in der Mark des babenbergischen Grafen Adalbert (der Siegreiche) nächst der Donau. Wie in mittelalterlichen Urkunden üblich, orientiert sich die Grenzziehung an topografischen Gegebenheiten; vgl. L. Mazakarini, Die Geschichte der Pfarre Groß-Enzersdorf, in: Stadt Groß-Enzersdorf. Beiträge zu ihrer Geschichte, Hrsg. Verein für Heimatkunde und Heimatpflege Groß-Enzersdorf, Bd. 3, Wien 1960, s. 5; F. Heller, Die Geschichte unserer Stadt Groß-Enzersdorf, Wien 1996, s. 29; H. Weigl, Histori- 
Wien im Marchfeld gelegen, zu schenken. In der Urkunde vom 14. November $1021^{3}$ heißt es, dass es sein Wille ist, seinem Bischof Egilbert von Freising (10061039), der lange Zeit Kanzler des Kaisers war, einen besonderen Beweis seiner Wertschätzung zu geben, da dessen Vorgänger, Bischof Hilto (810-835), das gestiftete Kloster Weihenstephan sehr am Herzen $\operatorname{lag}^{4}$. Das junge Stift Weihenstephan, gegründet im 8. Jahrhundert, konnte das weit entfernte Gebiet nicht verwalten und kultivieren. So kam es dann neun Jahre nach der Schenkung (1028) zu einem Tausch dieser Güter zwischen dem Kloster Weihenstephan (Benediktiner-Kloster) und Freising. Das Hochstift Freising übernahm das Land zwischen Donau und Wagram (eine durch die Donau gebildete Geländestufe) und gab dafür Gründe in Bayern an das Kloster Weihenstephan ab. Durch diesen Gütertausch erhielt die freisingische Herrschaft den zweiten Teil der Insel Sachsengang. Ursprünglich wollte das Kloster Weihenstephan eine Siedlung als Verwaltungszentrum anlegen, die unmittelbar bei Enzersdorf und Probstdorf liegen sollte. Aber erst mehr als 100 Jahre später ist Enzersdorf der Verwaltungssitz für den Besitz der Freisinger im Marchfeld geworden. Durch das Wirken der Freisinger bekam das Leben sowohl einen wirtschaftlichen als auch religiösen Aufschwung, sodass sich immer mehr Menschen ansiedelten. Neben dem herrschaftlichen Wirtschaftshof vor Ort

sches Ortsnamenbuch von Niederösterreich, Bd. 7, Wien 1975, U 40, Z 93; H. Glaser, Hrsg. Hochstift Freising. Beiträge zur Besitzgeschichte, München 1990, s.102, 276-277; Codex Diplomaticus Austriaco-Frisingensis. Sammlung von Urkunden und Urbaren zur Geschichte der ehemals freisingischen Besitzungen in Österreich, Hrsg. J. Zahn, Bd. 3, (zitiert als: Fontes rerum Austriacarum) 2/XXXI, Nr. 36, Wien 1871, s. 15. Der erste Teil des Namens der Insel Sahsonaganc verweist auf die Sachsen. Bekanntlich unterwarf Karl der Große die rebellischen Awaren, Sachsen und Bayern. „Ganc“ wiederum beschreibt die Stelle eines Flusses, an dem ein Übergang möglich war. Die Insel erhielt also den Namen von den Sachsen, die an einem Übergang der Donau siedelten. Der erwähnte Graf Adalbert vergrößerte das Gebiet seiner Mark insofern, als dass er das Marchfeld in seinen Herrschaftsbereich miteinbezog. Die Herrschaft der Babenberger begann in Österreich im Jahr 976. Leopold I. residierte in Melk, Leopold II. in Gars und Tulln und Leopold III. (der Heilige) in (Kloster-) Neuburg. Erst später wurde die Residenz nach Wien verlegt; vgl. Niederschrift der Tonaufzeichnung des Vortrages von Dr. Wolf Mazakarini, Freitag, 3. Mai 1996, s. 1-2; vgl. O. Schilder, Der politische Bezirk Gänserndorf in Wort und Bild, Gänserndorf 1970, s. 5; H. Schrom, 750 Jahre Pfarre Groß-Enzersdorf 1203-1953. Eine kleine Geschichte der Stadt und der Pfarre, Wien 1953, s. 11-12.

${ }^{3}$ Weitere vorhandene Urkunden über Groß-Enzersdorf finden sich in: Fontes rerum Austriacarum II, Nr. 36, s. 79-85, 106-107, 131-138, 160-162 und im Bayerischen Hauptstaatsarchiv, Hochstifsliteralien Freising 513. Den Urkundentext kann man auch auf lateinisch und deutsch finden in: H. Kovacic, Die Insel und die Herrschaft Sachsengang. Die frühe Genese der Herrschaften in Sachsengang und Groß-Enzersdorf und die Entstehung der dazu gehörenden Ortschaften, Saarbrücken 2014, s. 24-25, 129-130.

${ }^{4}$ Mazakarini, Die Geschichte der Pfarre Groß, s. 5. Hierzu, vgl. Schrom, Bilder aus dem Leben der Stadt, s. 5-31, in: Stadt Groß-Enzersdorf. Beiträge zu ihrer Geschichte, Hrsg. Verein für Heimatkunde und Heimatpflege Groß-Enzersdorf, Bd. 2, Wien 1960, s. 5; K. Brunner, Enzersdorf, die Hofmark der Bischöfe von Freising 1021-1803, in: Stadt Groß-Enzersdorf. Beiträge zu ihrer Geschichte, Hrsg. Verein für Heimatkunde und Heimatpflege Groß-Enzersdorf, Bd. 5, Wien 1965, S. 24-28; F. Goldmann, Groß-Enzersdorf. Politischer Bezirk Gänserndorf, in: Österreichisches Städtebuch 4, Niederösterreich: 1. Teil A-G, Wien 1988, s. 274; Urkundenbuch des Burgenlandes, Hrsg. I. Lindeck-Pozza, Bd. 4, Nr. 809, 1985. 
besa $ß$ das Hochstift auch einen bedeutenden Hof im Zentrum Wiens, den sogenannten Freisinger Hof am Graben 5 .

Es darf nicht vergessen werden, dass das Hochstift Freising in dieser Region nur die Grundherrschaft ausübte, es sich in kirchlichen Belangen aber mit dem Bistum Passau arrangieren musste. Neben dem Recht, den Pfarrer vorschlagen zu dürfen, gab es oft auch Streit über die Einhebung des Zehents (der zehnte Teil der Ernte) zwischen dem Bistum Passau und dem Hochstift Freising ${ }^{6}$.

Das Gebiet der Pfarre erstreckte sich bis zum Jahr 1749 bis Raasdorf mit Pysdorf im Norden, bis 1783 bis Wittau, Sachsengang, Ober-, Mittel-, und Unterhausen sowie nach Mühlleiten im Osten und bis 1946 bis Eßling, das heute innerhalb der Wiener Stadtgrenze liegt, im Westen. Die Jahreszahlen beziehen sich auf die jeweilige Pfarrerhebung bzw. Umstrukturierung. Wittau, Ober-, Mittel-, und Unterhausen wurden nach Probstdorf umgepfarrt. Interessant ist in diesem Zusammenhang, dass Enzersdorf selbst, als Filiale, der Mutterpfarre Stadlau parochia Stadelav zugehörig war. Stadlau gehörten 23 Ortschaften des südlichen Marchfelds an. Durch Witterungsbedingungen, wie Hochwässer und winterliche Eisstöße war die Kommunikation mit der Mutterpfarre oftmals stark eingeschränkt. Sogar die, durch die widrigen Bedingungen, zerstörte Pfarrkirche zum Heiligen Georg wurde von Stadlau nach Kagran (im Pfarrverzeichnis von 1429 wird Stadlau ecclesia hodie) verlegt. Aus den beschriebenen Gründen wurden nach und nach Ortschaften aus diesem Verband entlassen und konnten eigenständige Pfarren werden. Aus der Mutterpfarre Stadlau-Kagran sind die Pfarren Enzersdorf, Raasdorf, Deutsch-Wagram und Gerasdorf hervorgegangen?

Die erste Erwähnung in einem Urbar stammt aus dem Jahr 1160. Der Ort wird in der Quelle als Encinesdorf oder Enzeinesdorf bezeichnet ${ }^{8}$. Der bis heute erhaltene Name Enzersdorf bezieht sich in seinem Ursprung auf den Personennamen „Enzi“ oder „Engelschalk“, und dem Begriff „Dorf“, der „geschlossene bäuerliche Siedlung“ bedeutet. Im Laufe der Zeit wurde dann das Wort „Groß“ civitatis

${ }^{5}$ Niederschrift der Tonaufzeichnung, s. 1-3; vgl. Mazakarini, Die Geschichte der Pfarre Groß, s. 6; Heller, Die Geschichte unserer Stadt, s. 30; O. Schilder, Der politische Bezirk Gänserndorf in Wort und Bild, Gänserndorf 1970, s. 449; G. Holzmann, Groß-Enzersdorf und sein Lebensraum, Horn 1960, s. 25; O. Schilder, 950 Jahre Pfarrkirche St. Stephan in Probstdorf 1021-1971. Festschrift mit ortskundlichen Hinweisen über den Pfarrort Probstdorf mit seinen Filialorten Oberhausen, Schönau und Wittau, Horn 1971, s. 9; 86; S. Schwindshack1, Eßling und Groß-Enzersdorf im Wandel des 20. Jahrhunderts, Wolkersdorf 2009, s. 266; H. Wolf, Erläuterungen zum Historischen Atlas der österreichischen Alpenländer, II. Abt. Die Kirchen- und Grafschaftskarte 6. Teil: Niederösterreich, Wien 1955, s. 105; G. Pauser, Die Geschichte der Pfarre und Kirche St. Georg-Kagran, Dissertation an der Universität Wien 1994, s. 25-26. Mehr zum Thema Gütertausch zwischen Kloster Weihenstephan und Freising in: Kovacic, Die Insel und die, s. 26-28;101; 104; Fontes rerum Austriacarum II/XXXI, Nr. 15.

${ }^{6}$ Holzmann, Groß-Enzersdorf und sein Lebensraum, s. 12-13.

${ }^{7}$ O. Schilder, Raasdorf in Vergangenheit und Gegenwart, Deutsch-Wagram 1983, s. 125-126; vgl. Goldmann, Groß-Enzersdorf. Politischer Bezirk Gänserndorf, s. 282-283; Pauser, Die Geschichte der Pfarre und Kirche St. Georg-Kagran, s. 25-26.

${ }^{8}$ Niederschrift der Tonaufzeichnung, s. 2; vgl. Heller, Die Geschichte unserer Stadt, s. 30; Holzmann, Groß-Enzersdorf und sein Lebensraum, s. 9. 
maioris enczertorff hinzugefügt, um zur besseren Unterscheidung von anderen Ortschaften zu dienen, die den gleichen oder einen ähnlichen Namen tragen ${ }^{9}$. In dem genannten Ort befand sich schon die Verwaltung des Herrschaftsgebietes ${ }^{10}$. Wann eine erste Kirche in Groß-Enzersdorf errichtet bzw. gestiftet wurde, bleibt unklar, da zurzeit keine diesbezüglichen Quellen bekannt sind. Die erste Urkun$\mathrm{de}^{11}$, in der eine Kirche erwähnt wird, stammt aus dem Codex Lonsdorfianus ${ }^{12}$, einem Urkundenbuch, der die Rechte und den Besitzstand des Passauer Bistums erfasst. Darin findet sich der Hinweis, dass 1202/03 eine Capella, existierte. Zur damaligen Zeit war dies die Bezeichnung für eine kleine Kirche ${ }^{13}$. Die Entstehung der Pfarre ${ }^{14}$ ist eng mit dem wirtschaftlichen Leben des Marktes verbunden ${ }^{15}$.

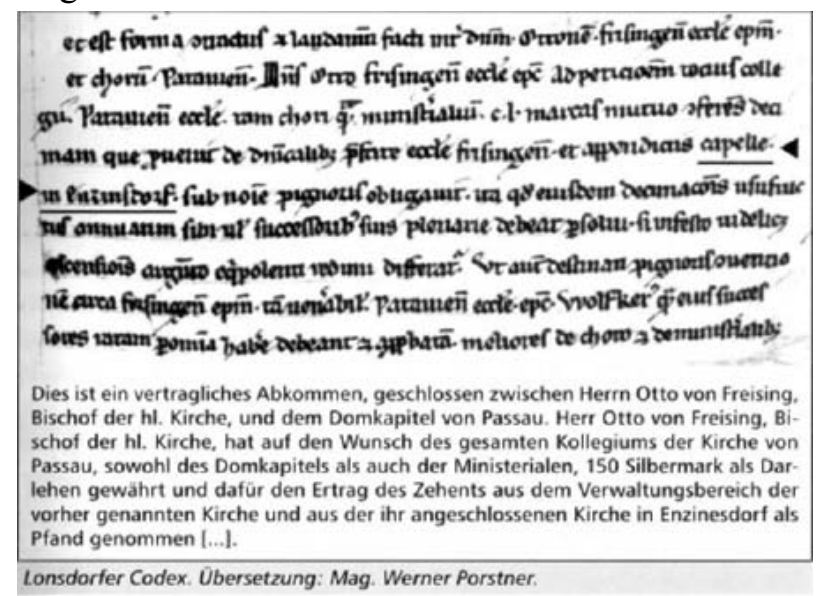

Il.1. Simum peris. Viverit. Scit. O tem, senatusus vis, cur anum quem mor aurs iam nonsula certantilnem deore, nes con dient, et etia sperbef

Weitere Hinweise zur Geschichte des Kirchengebäudes liefern diverse Kaufund Schenkungsvorgänge sowie Rechtsstreitigkeiten. Laut einer Urkunde lieh Bischof Otto II. von Freising dem Domkapitel von Passau wegen deren finanziellen Schwierigkeiten 150 Mark Silber. Dafür bekam er den Kirchenzehent von Enzers-

${ }^{9}$ Holzmann, Groß-Enzersdorf und sein Lebensraum, s. 10.

${ }^{10}$ Niederschrift der Tonaufzeichnung, s. 1-2.

${ }^{11}$ Urkunde Freising Nr. 18 im Bayerischen Hauptstaatsarchiv. Hierzu: M. Weltin, Ascherichsbrvgge - das Werden einer Stadt an der Grenze, in: Das Land und sein Recht, Ausgewählte Beiträge zur Verfassungsgeschichte Österreichs im Mittelalter, Wien-München 2006, s. 350-361.

${ }^{12}$ Umfasst Kopien von Urkunden, Rechte und die Besitztümer der Passauer Kirche. Geschrieben im Auftrag des Passauer Bischofs Otto von Lonsdorf.

${ }^{13}$ Niederschrift der Tonaufzeichnung, s. 1-2; vgl. Mazakarini, Die Geschichte der Pfarre Groß, s. 6; O. Schilder, Der politische Bezirk Gänserndorf in Wort und Bild, Gänserndorf 1970, s. 449; Heller, Die Geschichte unserer Stadt, s. 30; Kovacic, Die Insel und die, s. 113.

${ }^{14}$ In dieser Quelle ist freilich nicht die Rede von einer Pfarre parochia, sondern lediglich von einer Kapelle. Mit der Entwicklung des Ortes ist der Bau der Kirche sowohl als auch das religiöse Leben verbunden.

${ }^{15}$ Holzmann, Groß-Enzersdorf und sein Lebensraum, s. 12-13; vgl. Wolf, Erläuterungen zum Historischen Atlas der österreichischen, s. 105. 
dorf als Pfand ${ }^{16}$. Diese Urkunde berichtet über die Kirche nur sehr allgemein. Aus einer weiteren Freisinger Urkunde ist ersichtlich, dass eine kleine Kirche auf Freisinger Grund stand und vom ,Zugehör“ appendiciis der Priester erhalten wurde. Was noch das Hochstift Freising betrifft, so hat der Bischof das Vorschlagsrecht ius praesentationis, offene Stellen in Klöstern und Pfarren zu besetzen, egal in welcher Diözese sie liegen. Aus einer Urkunde aus dem Jahr 1212 bestätigt dies Manegolt, Bischof von Passau, dem Bischof von Freising hinsichtlich der Kirche in Enzersdorf ${ }^{17}$. Es ist aus einem Artikel des Heimatforschers Dr. Mazakarini zu schließen, dass spätestens im Jahre 1160 an der Kapelle bereits ein Priester tätig war, den das Bistum Freising ernennen durfte, obwohl zu dieser Zeit das ganze Gebiet der geistlichen Betreuung Passaus unterstand. Im Gegenzug erkannte Freising das Zehentrecht Passaus in der Herrschaft dieses Gebietes an. Bis heute ist allerdingst nicht sicher, ob in dieser Kapelle ein Pfarrer gewirkt hat. Man kann aber davon ausgehen, dass aus der genannten Capella eine Pfarrkirche entstand. Aus den Quellen weiß man allerdings nicht viel über dieses Gotteshaus. Der älteste Teil der an die Sakristei angebauten Turmkapelle hat sich in ihrem ursprünglichen Zustand erhalten. Die Rippen des Gewölbes weisen auf das erste Drittel des 13. Jahrhunderts hin.

Aus den Urkunden erfahren wir erst 1256 erstmals den Namen eines Pfarrers Leopold Liupoldus von Enzersdorf. Es ist aber anzunehmen, dass er nicht der erste Geistliche war, der hier wirkte. Aus den erhaltenen Protokollen geht hervor, dass der Pfarrer Leopold ein geachteter Mann war, dem man die Ausbildung eines Priesterstudenten anvertraut hatte. In den selben Protokollen wird ein Streit zwischen den Bistümern Freising und Passau über die Nachbesetzung der gut dotierten Pfarre Probstdorf geschildert, in dem er als geschworener Zeuge testis iuratus genannt wird. Passau favorisierte Dechant Wisinto und beharrte auf das Präsentationsrecht. Auf Grund dieses Umstandes hat das Bistum Freising gegenüber dem Bistum Passau opponiert, weil dies im Widerspruch zu den seinerzeit von Papst Innozenz II. an Bischof Otto I. von Freising erteilten Rechten stand. Umso mehr als der Bischof von Passau 1212 dem Bischof von Freising das Recht bestätigte, auf seinen Besitzungen das Vorschlagsrecht für die Pfarrer zu haben. Aus den genannten Protokollen, die seine Aussage enthalten, geht hervor, dass er seit zirka dreißig Jahren an der Kirche wirkte ${ }^{18}$. Pfarrer Leopold wird mehrmals in Urkunden erwähnt. Am 1. November 1265 erscheint er als Zeuge des Bischofs Konrad II. von Wittelsbach (1258-1279) bei der Erteilung der Genehmigung des Kaufes der Insel „Chleinwerde“ ${ }^{19}$. Zu dieser Zeit war Bischof Konrad II. in Enzersdorf anwesend, weil man die Genehmigung des Bischofs als Lehensherr be-

${ }^{16}$ Fontes rerum Austriacarum 2/XXXI, Nr. 124, ,..capelle in Enzinsdorf...“; vgl. Heller, Die Geschichte unserer Stadt, s. 33.

${ }^{17}$ Mazakarini, Die Geschichte der Pfarre Groß, s. 6; vgl. Fontes rerum Austriacarum 2/XXXI, Nr. 101, ,...sancimus etiam, ut in quocunque episcopatu in fundo Frisingensis ecclesie monasteria vel ecclesie edificate sint, assensu et consilio tuo in eis presbiteri statuantur".

${ }^{18}$ Ebenda, s. 6-8; vgl. Fontes rerum Austriacarum 2/XXXI, Nr. 184, s. 185-189; Kovacic, Die Insel und die, s. 90, 113; Heller, Die Geschichte unserer Stadt, s. 33.

${ }^{19}$ Ebenda, s. 9; vgl. Fontes rerum Austriacarum 2/XXXI, Nr. 251. 
nötigte, um die Insel „Chleinwerde“ zu kaufen. In einer anderen Urkunde wird Pfarrer Leopold als ,unser Treuer" bezeichnet - Leupoldus de Sachsengange fidelis noster. Dieses Schriftstück weist auch auf ein gutes Verhältnis des Pfarrers mit Bischof Konrad II. hin ${ }^{20} .1277$ wird Pfarrer Leopold als ...domino Liupoldo plebano in Entzesdorf... ${ }^{21}$ wieder genannt. Abermals ging es um einen Streit um die Pfarre Probstdorf. Am 17. Oktober desselben Jahres fuhr er im Auftrag des geistlichen Gerichts gemeinsam mit dem von Freising nominierten Probst Heinrich in die umstrittene Pfarre. Der geachtete Pfarrer wird am 31. Oktober als Zeuge einer bischöflichen Schenkung an den Probst Heinrich genannt ${ }^{22}$. Das letzte Mal wird Leopold ...Leupoldus plebanus de Enzinsdorf... in einer Urkunde vom 20. Mai 1281, eine Schenkung des Bischofs Friedrich von Freising (12781282) an das Kloster Lilienfeld ${ }^{23}$ betreffend, erwähnt. Als Pfarrer war er ungefähr fünfundfünfzig Jahre in Enzersdorf tätig. Vermutlich muss er achtzig Jahre alt gewesen sein. Über sein Schicksal fehlen uns weitere Informationen.

Ein anderer Priester erscheint in einer Urkunde vom 20. Februar 1308, die in Waidhofen an der Ybbs verfasst wurde, als „Herr Pfarrer Heinrich zu Entzestorf $^{\text {‘24 }}$. In einem Besitzstandsverzeichnis (Urbar) des Bistums Freising wird ein weiterer Pfarrer namentlich genannt, Bernhard. In derselben Quelle scheint sein Name öfters auf, letztmalig im Jahr 1317. Mehr wird über ihn nicht gesagt, wohl aber über seinen Bruder, der als balistarius, also „Armbrustschütze“, erwähnt wird. Dies lässt vermuten, dass die Brüder aus einer ansässigen Familie stammten. Der nächste Pfarrer taucht am 10. November 1332 in einer Verkaufsurkunde ${ }^{25}$, betreffend Weingärten, auf. Adressiert ist das Schriftstück an Friedrich Guetchint, Kastner $^{26}$ von Enzersdorf ${ }^{27}$. Im Besitzstandsverzeichnis von 1316 werden zwei Personen in Enczestorf genannt, von denen einer als Pfarrer „Leutpriester“ bezeichnet wird ${ }^{28}$.

Pfarrer Widenhofer hielt in seiner von Groß-Enzersdorf verfassten Geschichte (abgeschlossen am 28. November 1833) fest, dass beim letzten feindlichen Übergang der Franzosen über die Donau 1809 das Feuer alles vernichtete, sowohl herrschaftliche Dokumente als auch solche der Pfarre ${ }^{29}$.

Der Freisinger Bischof Berthold von Wehingen (1381-1410) ließ die Kirche im Stil der Gotik erweitern und dazu Material und Handwerker (Steinmetze) nach Enzersdorf kommen. Die Bauleute stammten vor allem aus Wien und ließen der

${ }^{20}$ Kovacic, Die Insel und die, s. 91; vgl. Fontes rerum Austriacarum 2/XXXI, Nr. 251, s. 268.

${ }^{21} \mathrm{Vgl}$. Fontes rerum Austriacarum 2/XXXI, Nr. 347.

${ }^{22}$ Mazakarini, Die Geschichte der Pfarre Groß, s. 10.

${ }^{23}$ Das Kloster Lilienfeld ist bis heute eine bestehende Zisterzienser Abtei in Niederösterreich, in der Nähe von St. Pölten.

${ }^{24} \mathrm{Vgl}$. Fontes rerum Austriacarum 2/XXXI, Nr. 473.

${ }^{25}$ Ebenda, 2/XXXI, Nr. 611.

${ }^{26}$ Inhaber des Kastenamts (Kasten, Kammer), der die Einkünfte des Grundherrn verwaltet.

${ }^{27}$ Mazakarini, Die Geschichte der Pfarre Groß, s. 10.

${ }^{28}$ Kovacic, Die Insel und die, s. 106.

${ }^{29}$ J. Wiedenhofer, Geschichte der Stadt Groß-Enzersdorf, Pagina 170, 1828. Wiedenhofer Johann war Pfarrer in Groß-Enzersdorf von 1822 bis 1846 und verfasste eine Geschichte der Stadt. 
Kirche ähnliche Sorgfalt angedeihen ${ }^{30}$ wie dem Dom zu St. Stephan. Bis heute ist der gotische Chor mit Kreuzrippengewölbe erhalten geblieben, der auf eine Stiftung Bischof Bertholds zurückgeht ${ }^{31}$. Es gilt noch, auf das von den österreichischen Herzögen Wilhelm und Leopold verliehene Stadtrecht hinzuweisen. Es erlaubte ihrem Kanzler Berthold von Wehingen, gleichzeitig Bischof von Freising und Grundherr der freisingischen Besitzungen im Marchfeld, am 4. April 1396 den Markt Enzersdorf mit einer mächtigen Ringmauer umgeben zu dürfen ${ }^{32}$. Die Einnahmen aus Marktrecht und Landgericht für den Enzersdorfer Besitz gehen auf die Bestätigung Kaiser Friedrich I genannt Barbarossa zurück und mussten nicht an den Landesherrn abgeführt werden. Schrittweise gelang es den Bischöfen von Freising, ihre Grundherrschaft mit weiteren Rechten auszustatten. Um 1277 war die Herrschaft Enzersdorf mit allen wesentlichen Herrschaftsrechten versehen. Bischof Berthold erreichte im Jahr 1396 für Enzersdorf die Stadterhebung ${ }^{33}$.

Mit der Erlangung des Stadtrechtes ${ }^{34}$ erhielt die Stadt auch ihr Stadtwappen. In der oberen Hälfte sieht man einen Mohrenkopf Aethiopis Frisingensis auf einer goldenen Zickzacklinie auf blauem Grund und in der unteren Hälfte das Wappen des Geschlechts der Wehinger, der Familie des Freisinger Bischofs Berthold, mit gezogenem Balken auf blauem Grund ${ }^{35}$. Vom 12. Jahrhundert bis 1803 blieb Enzersdorf der Hauptort des Hochstiftes Freising im Marchfeld ${ }^{36}$.

Der Autor des Artikels bezieht sich nur auf die Literatur, die von Heimatforschern veröffentlicht wurde. Es ist wahrscheinlich noch mehr in Freising und München, dieses Thema betreffend, zu erforschen. Es bleiben noch viele Fragen zur Entstehung der Pfarre und den hier wirkenden Priestern offen. Wie schon im

${ }^{30}$ Heller, Die Geschichte unserer Stadt, s. 38; 40; vgl. L. Winkler, Stadt Groß-Enzersdorf. Beiträge zu ihrer Geschichte, Bd. 1, Wien 1960, s. 10.

${ }^{31}$ Schrom, 750 Jahre Pfarre, s. 33.

${ }^{32}$ Nach neuesten archäologischen und historischen Erkenntnissen existierte aber bereits eine Marktmauer, die zwischen 1396-1399 lediglich aufgezont wurde. Hauptstaatsarchiv München: Hochstift Freising, Urkunde v. 1396, IV, 4; vgl. Holzmann, Groß-Enzersdorf und sein Lebensraum, s. 14; Schrom, 750 Jahre Pfarre, s. 14-16.

${ }^{33}$ H. Glaser Hrsg., Hochstift Freising. Beiträge zur Besitzgeschichte, München 1990, s. 102; 281; vgl. Mazakarini, Die Geschichte der Pfarre, s. 5; Kovacic, Die Insel und die, s. 48-51.

${ }^{34} \mathrm{Mehr}$ zu diesem Thema bei E. Klebel, Die Städte und Märkte des bayerischen Stammesgebietes in der Siedlungsgeschichte, Zeitschrift für bayerische Landesgeschichte 12, München 1939/1940, s. 37-93.

${ }^{35}$ An einem der drei Stadttore erinnert eine Inschrift und das Wappen an die Stadtwerdung; vgl. Holzmann, Groß-Enzersdorf und sein Lebensraum, s. 14. Die eingemeißelte Inschrift war lange Zeit unleserlich. Der lateinische Text lautet: M TER C NOVEM DECIES ANNO QUOQE NONO HOS CIVES VEROS MURO CINGEBAT HERES PERCHTOLT DE WECHING GNATUS CAPUT QUOQUE FREYSING. Übersetzung: Im Jahr Eintausend dreimal Hundert neunmal Zehn und Neun umgab diese treuen Stadtbürger mit einer Mauer Perchtolt geborener Erbe von Wehingen und Freysings Oberhaupt. Die Bewohner der Stadt werden bereits cives genannt, Bürger einer civitas; vgl. L. Winkler, Berthold von Wehingen, Bischof von Freising, österreichischer Kanzler und Groß-Enzersdorfs erster Stadtherr, in: Stadt Groß-Enzersdorf. Beiträge zu ihrer Geschichte, Hrsg. Verein für Heimatkunde und Heimatpflege Groß-Enzersdorf, Bd. 1, Wien 1960, s. 12-15.

${ }^{36}$ Mazakarini, Die Geschichte der Pfarre, s. 39. 
Artikel erwähnt, wurden während der Napoleonischen Kriege die Unterlagen durch ein Feuer vernichtet. Die freisingische Herrschaft der Bischöfe im Marchfeld hat die Entwicklung von Groß-Enzersdorf, wie auch von Raasdorf, Probstdorf und anderen Ortschaften geprägt. Damals hat die Politik die kirchlichen Einrichtungen beeinflusst und umgekehrt. Die Quellenedition Fontes rerum Austriacarum erlaubt uns einen besseren Blick auf frühere Zeiten und macht uns noch besser mit der Herrschaft der Bischöfe von Freising in Österreich vertraut.

Das Wappen von Bischof Berthold von Wehingen ist bis heute das Stadtwappen von Groß-Enzersdorf geblieben. Dem Bischof ist auch der Platz auf der Südseite gewidmet - an der Hauptfassade der Kirche ist sein Wappen in einem Kirchenfenster verewigt. 2017 wurden sechs Messgewänder der Pfarre „Maria Schutz“ gestiftet, auf deren Rückseiten das Wehinger- oder Stadtwappen zu sehen ist.

\section{BIBLIOGRAFIE}

\section{Quellenverzeichnis}

Codex Diplomaticus Austriaco-Frisingensis. Sammlung von Urkunden und Urbaren zur Geschichte der ehemals freisingischen Besitzungen in Österreich, Hrsg. Zahn J., (=Fontes rerum Austriacarum) 2: Diplomataria et acta. Band XXXI, Wien 1871, S. 79-85, 106-107, 131-138, 160-162.

Hauptstaatsarchiv München: Hochstift Freising, Urkunde V. 1396, IV, 4.

Urkunde Freising Nr. 18 im Bayerischen Hauptstaatsarchiv.

Urkundenbuch des Burgenlandes, Lindeck-Pozza I. Hrsg., Band 4, 1985.

\section{Literaturverzeichnis}

Goldmann Friederike, Groß-Enzersdorf. Politischer Bezirk Gänserndorf, in: Österreichisches Städtebuch 4, Niederösterreich: 1. Teil A-G, Wien 1988, s. 271-285.

Heller Friedrich, Die Geschichte unserer Stadt Groß-Enzersdorf, Wien 1996.

Holzmann Gustav, Groß-Enzersdorf und sein Lebensraum, Horn 1960.

Klebel Ernst, Die Städte und Märkte des bayerischen Stammesgebietes in der Siedlungsgeschichte, „Zeitschrift für Bayerische Landesgeschichte“, 12 (1939/1940) s. 37-93.

Kovacic Herbert, Die Insel und die Herrschaft Sachsengang. Die frühe Genese der Herrschaften in Sachsengang und Groß-Enzersdorf und die Entstehung der dazu gehörenden Ortschaften, Saarbrücken 2014.

Mazakarini Leopold, Die Geschichte der Pfarre Groß-Enzersdorf, in: Stadt Groß-Enzersdorf. Beiträge zu ihrer Geschichte, Hrsg. Verein für Heimatkunde und Heimatpflege Groß-Enzersdorf, Bd. 3, Wien 1960, s. 5-63.

Niederschrift der Tonaufzeichnung des Vortrages von Dr. Wolf Mazakarini, Freitag, 3. Mai 1996.

Pauser Georg, Die Geschichte der Pfarre und Kirche St. Georg-Kagran, Dissertation an der Universität Wien 1994.

Schilder Otto, Der politische Bezirk Gänserndorf in Wort und Bild, Gänserndorf 1970.

Schilder Otto, 950 Jahre Pfarrkirche St. Stephan in Probstdorf 1021-1971. Festschrift mit ortskundlichen Hinweisen über den Pfarrort Probstdorf mit seinen Filialorten Oberhausen, Schönau und Wittau, Horn 1971. 
Schilder Otto, Raasdorf in Vergangenheit und Gegenwart, Deutsch-Wagram 1983.

Schrom Hermann, 750 Jahre Pfarre Groß-Enzersdorf 1203-1953. Eine kleine Geschichte der Stadt und der Pfarre, Wien 1953.

Schrom Hermann, Bilder aus dem Leben der Stadt, in: Stadt Groß-Enzersdorf. Beiträge zu ihrer Geschichte, Hrsg. Verein für Heimatkunde und Heimatpflege Groß-Enzersdorf, Bd. 2, Wien 1960, s. 5-31.

Schwindshackl Sophie, Eßling und Groß-Enzersdorf im Wandel des 20. Jahrhunderts, Wolkersdorf 2009.

Weltin Max, Ascherichsbrvgge - das Werden einer Stadt an der Grenze, in: Das Land und sein Recht, Ausgewählte Beiträge zur Verfassungsgeschichte Österreichs im Mittelalter, Wien-München 2006, s. 338-374.

Wiedenhofer J., Geschichte der Stadt Groß-Enzersdorf, 1828.

Winkler Leopold, Berthold von Wehingen, Bischof von Freising, österreichischer Kanzler und Groß-Enzersdorfs erster Stadtherr, in: Stadt Groß-Enzersdorf. Beiträge zu ihrer Geschichte, Hrsg. Verein für Heimatkunde und Heimatpflege Groß-Enzersdorf, Bd. 1, Wien 1960, s. 5-19.

Wolf Hans, Erläuterungen zum Historischen Atlas der österreichischen Alpenländer, II. Abteilung, Die Kirchen- und Grafschaftskarte; 6. Teil: Niederösterreich, Wien 1955.

\section{BISKUPSTWO FREISING, A KSZTALTOWANIE SIE ŻYCIA PARAFIALNEGO W GROSS-ENZERSDORF AŻ DO DZIALALNOŚCI BISKUPA BERTHOLDA Z WEHINGEN}

\section{Streszczenie}

Temat artykułu nawiązuje do początku panowania biskupów z Freising (Bayern) na południe od rzeki Dunaj, w miejscowości Groß-Enzersdorf, w regonie zwanym Marchfeld, i ich wpływu na życie religijne. Problematyka ta sięga czasów cesarza Henryka II, który w dokumencie z 14 listopada 1021 r. przekazał benedykteńskiemu klasztorowi Weihenstephan na własność część wyspy (łac. insula Sahsonaganc), ramię Dunaju w pobliżu Wiednia. Jednak w krótkim czasie doszło do wymiany dóbr pomiędzy klasztorem Weihenstephan a biskupstwem Freising - na jego własność przeszła druga część wyspy Sachsengang oraz dobra ziemskie w tym regionie.

Pierwsza wzmianka nazwy miejscowości, mianowicie Enzinesdorf albo Enzeinesdorf, pojawiła się w dokumencie (Urbar; Besitzrechtsverzeichnis) z 1160 r. Warto zauważyć, że przedrostek Groß (duży, wielki) został dodany później, aby odróżnić miejscowości o tej samej nazwie. Brakuje informacji o budowie pierwszej kaplicy w tej miejscowości, jednakże Codex Lonsdorfianus obejmujący księgi datowane na rok 1202/1203 zawiera wzmiankę na temat drewnianego budynku Capella, co oznaczało w tamtym czasie mały kościół. W dostępnej literaturze opisującej ten okres jest mowa o kościele, a nie o parafii, więc dokładny czas erygowania (łac. erigo) nie jest znany. Przyjmuje się jednak, że na miejscu owej budowli został w późniejszym czasie wzniesiony kościół parafialny. Wiadomo też, że najstarsza zachowana część pochodzi z XIII wieku. Kwestia początków budowy świątyni pozostawia otwarte pytania i daje możliwość dalszych badań. 
Należy wspomnieć, iż parafia Groß-Enzersdorf ma swoje korzenie w parafii Stadlau-Kagran, która w tamtym czasie obejmowała 23 miejscowości. Z niej wywodzą się również parafie Raasdorf, Deutsch-Wagram i w późniejszym czasie - Gerasdorf. Pierwsza wzmianka na temat duchownego o imieniu Leopold w Enzersdorf pochodzi z 1256 r., jest on wymieniony jako świadek w sporze pomiędzy biskupstwami Freising i Passau o prawo do dóbr, zysków i patronatu, jak i prawa do obsadzania zamożnej parafii Probstdorf. Jego imię pojawia się jeszcze kilka razy w dokumentach. Innym wspomnianym duchownym jest proboszcz Henryk. Szczegóły, oprócz jego imienia, nie są znane. Interesującą kwestią jest to, że duchowny został wymieniony wraz ze swoim bratem, który pełnił na zamku w Enzersdorf funkcję kusznika, co pozwala na przypuszczenie, że pochodzili z owej miejscowości. Nie są znane bliższe fakty historyczne z życia i działalności pracujących w parafii w tym okresie księży, jak i z zakresu życia religijnego.

Biskup Freising Bertold von Wehingen, któremu miejscowość wiele zawdzięcza, rozpoczął rozbudowę kościoła w stylu gotyckim. Mając przywilej „targowy” (łac. ius fori, niem. Marktrecht), wybudował mur obronny i pozostawił po sobie herb, który do dziś jest herbem miasta.

Jak wspomina proboszcz Widenhofer w swojej Historii, ukończonej w 1833 r., wszelkie dokumenty, zapiski, jak i kroniki parafialne uległy zniszczeniu (spaleniu) w trakcie oblężenia przez armię cesarza Napoleona w 1809 r. Panowanie biskupów z Freising przekładało się na rozwój życia w Enzersdorf zarówno gospodarczego, społecznego, jak i religijnego. Trzeba też wspomnieć, że region Marchfeldu w zarządzaniu dobrami ziemskimi był pod wpływem biskupstwa Freising, natomiast co do jurysdykcji kościelnej - Passau. $\mathrm{Na}$ tym gruncie dochodziło do sporów, których ślady znajdujemy w różnych dokumentach na temat miejscowości i życia religijnego.

Powyższy artykuł opiera się na dostępnej literaturze miejscowej i przedstawia zarys początków powstania życia religijnego i parafii pod rządami biskupów z Freising.

Słowa kluczowe: proboszcz; parafia; archidiecezja wiedeńska;

Groß-Enzersdorf; biskup Berthold von Wehingen; Hochstift; Freising; Pasawa; Weihenstephan; certyfikat; Leopold; Kagran 


\title{
THE FREISING DIOCESE AND THE DEVELOPMENT OF PARISH LIFE IN GROSS-ENZERSDORF BEFORE THE INSTALLATION OF BISHOP BERTHOLD OF WEHINGEN
}

\begin{abstract}
The article explores the beginnings of the rule of Freising bishops in Bayern, south of the Danube River, in Groß-Enzersdorf, in the region called Marchfeld, and their influence on religious life in the region. The problem in question dates back to Henry II, Holy Roman Emperor, who handed over the ownership of a part of the Sachsengang island (Lat. insula Sahsonaganc), an arm of the Danube near Vienna, to the Benedictine monastery Weihenstephan in a document of 14 November, 1021. Soon, however, the Weihenstephan monastery exchanged the property with the bishopric of Freising, which became the owner the other part of the Sachsengang island and the land goods in this region.
\end{abstract}

The name of the place - Enzinesdorf or Enzeinesdorf - first appeared in a document of 1160 (Urbar; Besitzrechtsverzeichnis). It is worth noting that the Groß prefix (large or big) was only added later to distinguish between towns of the same name. There is no information regarding the construction of the first chapel in this locality. The Codex Lonsdorfianus covering books dated 1202/1203, however, mentions a wooden Capella building, which meant a small church at that time. It is assumed that a parish church was erected on the site of this building at a later point. What is also known is that its oldest preserved part goes back to the 13th century.

It is important to note that the Groß-Enzersdorf parish is rooted in the parish of Stadlau-Kagran, which covered 23 towns and villages at that time. A reference to a clergyman of Enzersdorf first appeared in 1256. A man named Leopold was indicated as a witness in a dispute between the Freising and Passau dioceses over the right to goods, profits and patronage as well as the right to appoint priests to the parish of Probstdorf. Another priest mentioned is the documents is the parish priest named Henry. Interestingly enough, the clergyman appears in the text together with his brother, who served as a crossbowman at the Enzersdorf castle. This would suggest that they came from that town.

Bertold von Wehingen, the Freising Bishop, initiated the construction works to expand the church and renovate it in the Gothic style. He also had a defensive wall built. The coat of arms he left behind is still used as the coat of arms of the city.

According to the "History" of the parish priest Widenhofer (1833), the parish archives were destroyed (burned) during a Napoleon's siege in 1809. The reign of the bishops of Freising inspired the development of economic, social and religious life in Enzersdorf. It should also be mentioned that the entire Marchfeld region was under the influence of the Freising bishopric in terms of the land management while it remained under the ecclesiastical jurisdiction of Passau. This led to numerous disputes, traces of which can be found in various documents regarding the place and its religious life.

Keywords: parish priest; parish; Archdiocese of Vienna; Groß-Enzersdorf; Bishop Berthold von Wehingen; Hochstift; Freising; Passau; Weihenstephan; certificate; Leopold; Kagran 